\title{
Functional outcome after sacrospinous hysteropexy for uterine descensus
}

\author{
Viviane Dietz • Marieke Huisman • Joyce M. de Jong • \\ Peter M. Heintz • Carl H. van der Vaart
}

Received: 21 September 2007 / Accepted: 14 November 2007 / Published online: 23 February 2008

(C) The Author(s) 2008

\begin{abstract}
The study aimed to evaluate urogenital symptoms, defecatory symptoms and quality of life before and after a sacrospinous hysteropexy for uterovaginal prolapse. Seventy-two women with symptomatic uterovaginal prolapse were treated with sacrospinous hysteropexy. Before and after surgery, urogenital and defecatory symptoms and quality of life were assessed with a validated questionnaire. Anatomical outcome was assessed by means of pelvic examination before and after surgery. The mean follow-up time was 12.7 months. Scores on all domains of urogenital symptoms and defecatory symptoms, except for the pain and fecal incontinence domain, improved significantly. Also, quality of life improved on all domains. No major complications were encountered.
\end{abstract}

Keywords Pelvic organ prolapse - Sacrospinous hysteropexy · Functional outcome · Quality of life

\section{Introduction}

In the current debate on the optimal surgical treatment of a uterine descent, several vaginal and abdominal techniques have been described. In case of a vaginal vault prolapse, the sacrospinous ligament fixation has proven to be an effective treatment [1]. The sacrospinous ligament fixation can also be performed as primary treatment for a uterine descent, a technique that can be referred to as 'sacrospinous hyster-

V. Dietz $(\bowtie) \cdot$ M. Huisman $\cdot$ J. M. de Jong $\cdot$

P. M. Heintz $\cdot$ C. H. van der Vaart

Department of Perinatology and Gynecology,

University Medical Center Utrecht,

Room F05.126, Heidelberglaan 100, P.O. Box 85500,

3508 GA Utrecht, The Netherlands

e-mail: viviane.dietz@sapio.nl opexy'. This procedure has been described in women who wanted to preserve the uterus to retain fertility $[2,3]$. Several studies have shown that the sacrospinous hysteropexy is anatomical efficient and safe and most women are highly satisfied about the procedure [4-10]. Outcome in these studies was mainly assessed in terms of anatomical results, and the majority of these studies did not evaluate urogenital symptoms and quality of life with validated questionnaires. So, although anatomical outcome of the sacrospinous hysteropexy appears to be good, we cannot conclude from current literature that this type of surgery is associated with a significant functional improvement of urogenital and defecatory symptoms. Measuring this functional outcome preoperatively and postoperatively was one of the recommendations for future research from a recent review on the subject [11]. The main objective of this study was to assess urogenital and defecatory symptoms and quality of life before and after sacrospinous hysteropexy. Secondary, we assessed anatomical outcome.

\section{Materials and methods}

Patients

In the period between December 2001 and April 2005, 72 women underwent a sacrospinous hysteropexy at the University Medical Center Utrecht, The Netherlands.

A woman was eligible for participation if she had a symptomatic pelvic organ prolapse and wanted surgical correction with preservation of the uterus. Exclusion criteria were abnormal uterus or ovaries on ultrasound examination, abnormal bleeding pattern and abnormal cervical cytology. All women answered a standardized questionnaire covering urogenital symptoms, defecatory symptoms and quality of 
life before and after surgery. Urodynamic assessment was performed in all women preoperatively. We did not try to diagnose occult stress incontinence. The study was approved by the local ethics committee.

\section{Surgery}

Surgery was performed by two experienced surgeons who at least had performed 20 sacrospinous hysteropexies before start of the study. The sacrospinous hysteropexy was performed unilaterally to the right ligament. A midline incision in the posterior vaginal wall is extended to the posterior part of the cervix. Through sharp and blunt dissection, the right sacrospinous ligament is made visible with the use of three Breisky retractors. Two nonabsorbable Prolene ${ }^{\circledR}$ sutures (Ethicon, Sommerville, NJ, USA) are placed through the right sacrospinous ligament, approximately $2 \mathrm{~cm}$ median to the ischial spine, and subsequently placed through the posterior side of the cervix in the midline. An additional anterior and/or posterior colporrhaphy (fascia plication), with absorbable Vicryl ${ }^{\circledR} 2$ 0 interrupted sutures (Ethicon, Sommerville, NJ, USA), was performed when indicated by the judgment of the individual gynecologist. In case of stress urinary incontinence, confirmed by urodynamic tests, a surgical procedure of the Tension-free Vaginal Tape (TVT, Ethicon, Sommerville, NJ, USA) was performed as described by Ulmsten [12]. All women received peri-operative thrombosis prophylaxis (anti-Xa) and a single dose of intravenous prophylactic antibiotic (amoxicillin/clavunalic acid). Post-operatively, a 14-French Foley indwelling bladder catheter with a 5-ml balloon was placed in all women and removed after 1 day (in case of an additional anterior colporrhaphy after 3 days).

\section{Measurements}

Data collection took place in October/September 2005, at least 3 months after surgery of the last participating woman. The following data were collected: age at the time of surgery; medical history; hospital stay and perioperative and postoperative complications. The anatomical outcome of all women was assessed with the Pelvic Organ Prolapse Quantification score (POP-Q) [13], which is described by the International Continence Society as a reliable and specific method to measure the pelvic organ support. Before surgery, POP-Q was performed by one of the two surgeons. After surgery, POP-Q was performed by one of three independent investigators. In the analysis, we dichotomized the POP-Q stage of prolapse into stage 1 or less and stage 2 or higher. Although we know that women with pelvic organ prolapse experience symptoms that do not necessarily correlate with the severity of prolapse, we have chosen this approach to try to separate potential clinical irrelevant from clinical relevant recurrences [14].

Urogenital symptoms were measured before and after surgery with a standardized questionnaire, the Urogenital Distress Inventory (UDI), which has been validated for the Dutch population [15]. In this validation study on a large population-based sample, it was shown that the domain construction of the Dutch version was different from the original one. The following five domains were identified: urinary incontinence, overactive bladder, pain, obstructive micturition and prolapse. The scores of these domains vary between 0 and 100. A high score on a particular domain indicates more bothersome symptoms. The incidence of urinary incontinence before surgery was measured as follows: a woman was considered to have stress urinary incontinence if she replied positively to the question "Do you experience urinary leakage related to physical activity, coughing or sneezing?". Urge urinary incontinence was scored if the question, "do you experience urinary leakage related to the feeling of urgency?" was answered positively.

All patients completed a questionnaire, the Defecatory Distress Inventory (DDI) to assess the presence of defecation symptoms before and after surgery. This questionnaire was developed by our research group to assess the presence of defecation symptoms [16]. The DDI consists of 15 items about symptoms related to obstructive defecation, constipation, fecal incontinence and pain related to defecation. The questions were developed after studying the literature and international definitions, interviewing patients who presented with constipation or fecal incontinence, and by interviewing three experts in the field from the Department of Surgery and Department of Obstetrics and Gynecology from the University Medical Center Utrecht, The Netherlands. Eventually, a structured interview of the 15 selected items was held with 20 female patients. The DDI was used as, at present, there are no other Dutch validated questionnaires to measure quality of life related to defecation symptoms. The design on the questions is identical to those of the UDI with domain scores between 0 and 100. Again, a high score on a particular domain indicates more bothersome symptoms. The DDI was used in previous studies at our department [4, 9, 17].

Before and after surgery, disease-specific quality of life was measured with the Incontinence Impact Questionnaire (IIQ), validated for the Dutch population [15]. These questions cover the following five domains: physical functioning, mobility, emotional functioning, social functioning and embarrassment. The score ranged between 0 (best quality of life) and 100 (worst quality of life).

Effect sizes were measured as a useful way to estimate whether an improvement on a particular domain was 
considered to have a small, moderate or large clinical relevance.

\section{Statistical analysis}

Descriptive statistics were used for the whole population. To compare scores on urogenital and defecatory symptoms before and after surgery a paired samples $t$ test was used. The significance level was set at $\alpha$ of 0.05 . The effect size was calculated by Cohen's $d$ test which is defined as the difference between two means divided by the pooled standard deviation for those means [18]. An effect size of 0.2 was considered to be small, 0.5 to be median and 0.8 or higher to be large [18]. Statistical analysis was performed in SPSS 12.0 for Windows.

\section{Results}

Table 1 shows the characteristics of the 72 women. The fast majority had a combination of sacrospinous hysteropexy with an anterior colporrhaphy $(87.5 \%)$. Five women $(6.9 \%)$

Table 1 Patient characteristics

\begin{tabular}{|c|c|}
\hline Patient characteristics & $n=72$ \\
\hline Age $(\text { years })^{\mathrm{a}}$ & $57.2(11.9)$ \\
\hline Surgery for prolapse in medical history & $3(4.2)$ \\
\hline $\begin{array}{l}\text { Surgery for urinary incontinence in } \\
\text { medical history }\end{array}$ & $4(5.6)$ \\
\hline Urinary incontinence before surgery ${ }^{\mathrm{b}}$ & $40(55.6)$ \\
\hline Urge incontinence & $9(12.5)$ \\
\hline Stress incontinence & $21(29.2)$ \\
\hline Combination stress and urge incontinence & $10(13.9)$ \\
\hline \multicolumn{2}{|l|}{ Gynecological examination before surgery } \\
\hline Descensus uteri stage 2 or more & $67(93.2)$ \\
\hline Cystoceles stage 2 or more & $57(79.2)$ \\
\hline Rectoceles stage 2 or more & $19(26.4)$ \\
\hline Enterocele & $1(1.4)$ \\
\hline \multicolumn{2}{|l|}{ Surgery } \\
\hline Sacrospinous hysteropexy & 8 (11.1) \\
\hline $\begin{array}{l}\text { Sacrospinous hysteropexy }+ \text { anterior } \\
\text { colporrhaphy }\end{array}$ & $54(75)$ \\
\hline $\begin{array}{l}\text { Sacrospinous hysteropexy }+ \text { anterior } \\
\text { and posterior colporrhaphy }\end{array}$ & $9(12.5)$ \\
\hline $\begin{array}{l}\text { Sacrospinous hysteropexy }+ \text { posterior } \\
\text { colporrhaphy }\end{array}$ & $1(1.4)$ \\
\hline Additional TVT & $15(20.8)$ \\
\hline Hospital stay $\left(\right.$ days) ${ }^{\mathrm{c}}$ & $3.5(1-8)$ \\
\hline Follow-up in months ${ }^{\mathrm{c}}$ & $12.7(3-33)$ \\
\hline
\end{tabular}

had a sacrospinous hysteropexy of a stage 1 uterine descent. In these women, the surgeon decided to perform a sacrospinous hysteropexy during surgery because the uterine descent under anaesthesia was stage 2 . The mean follow-up time was 12.7 months (median 11 months).

Perioperative and postoperative complications are shown in Table 2. One woman needed repeated surgery for postoperative bleeding a couple of hours after the primary procedure. Total blood loss was estimated at $400 \mathrm{cc}$. There were no incidental bladder or rectal injuries. Of the 20 women $(27.8 \%)$ who had urinary retention over $100 \mathrm{ml}$ after removal of the indwelling bladder catheter, five women $(6.9 \%)$ needed intermittent self-catheterisation more than 2 weeks after surgery. However, at 4 weeks, no women had significant urinary retention. This complication only occurred in women who underwent an anterior colporrhaphy. Seven women with cystitis received antibiotics and made an uneventful recovery. Five women (6.9\%) developed right-sided buttock pain which persisted longer than 2 weeks. It resolved spontaneously within 6 weeks. No removal of the sacrospinal suture was required.

During the follow-up period, a total of 16 women $(22.2 \%)$ had a recurrent prolapse of one of the compartments. Five women $(6.9 \%)$ had a recurrent prolapse of the uterus (four women stage 2, one woman stage 3). Ten women $(13.9 \%)$ had a cystocele stage 2 or more (eight women stage 2, two women stage 3) and two women $(2.8 \%)$ had a prolapse of the posterior compartment stage 2 . All the women with a recurrent cystocele had had surgery of the anterior compartment combined with the hysteropexy, so there were no de novo cystoceles. The two women with a recurrent rectocele did not have surgery of the posterior compartment combined with the sacrospinous hysteropexy, so these can be considered as de novo rectoceles.

Table 2 Complications related to surgery

\begin{tabular}{ll}
\hline Patient characteristics & $n=72$ \\
\hline Complications during surgery & $0(0)$ \\
Complications after surgery & $32(44.4)$ \\
Second surgery because of bleeding & $1(1.4)$ \\
Buttock pain & $13(18.1)$ \\
Buttock pain $<2$ weeks & $8(11.1)$ \\
Buttock pain $>2$ weeks & $5(6.9)$ \\
Vaginal hematoma & $2(2.8)$ \\
Urinary tract infection & $7(9.7)$ \\
Retention bladder & $20(27.8)$ \\
Bladder catheterisation $<2$ weeks & $15(20.8)$ \\
Bladder catheterisation $>2$ weeks & $5(6.9)$ \\
Vaginal adhesion & $3(4.2)$ \\
\hline
\end{tabular}

Data are numbers $(\%)$ 
Table 3 Urinary Distress Inventory (UDI), Defecatory Distress Inventory (DDI) and Incontinence Impact Questionnaire (IIQ)
Effect size: $0.2=$ small effect, $0.5=$ median effect, $0.8=$ large effect

${ }^{\mathrm{a}}$ Mean (standard deviation)

\begin{tabular}{lllll}
\hline & Before surgery $^{\mathrm{a}}(n=72)$ & After surgery $^{\mathrm{a}}(n=72)$ & $P$ value & Effect size \\
\hline UDI & & & & \\
Urinary incontinence & $18.5(24.2)$ & $10.5(21.0)$ & 0.012 & 0.35 \\
Overactive bladder & $29.5(26.7)$ & $14.4(18.6)$ & 0.000 & 0.66 \\
Pain & $30.1(26.7)$ & $9.5(16.7)$ & 0.000 & 0.92 \\
Obstructive micturition & $27.0(28.3)$ & $10.8(19.7)$ & 0.000 & 0.66 \\
Genital prolapse & $56.6(32.0)$ & $5.6(17.4)$ & 0.000 & 2.0 \\
DDI & & & & \\
Constipation & $11.9(18.8)$ & $6.3(9.8)$ & 0.021 & 0.37 \\
Obstructive defecation & $13.3(19.7)$ & $8.3(11.8)$ & 0.016 & 0.22 \\
Pain & $4.5(16.3)$ & $2.8(8.3)$ & 0.498 & 0.13 \\
Fecal incontinence & $8.6(20.7)$ & $8.5(14.4)$ & 0.955 & 0.01 \\
IIQ & & & & \\
Physical functioning & $26.0(24.4)$ & $9.4(15.1)$ & 0.000 & 0.82 \\
Mobility & $25.0(22.8)$ & $12.6(18.4)$ & 0.000 & 0.60 \\
Emotional health & $23.2(23.3)$ & $8.0(14.2)$ & 0.000 & 0.79 \\
Social functioning & $14.8(19.1)$ & $4.5(11.8)$ & 0.000 & 0.65 \\
Embarrassment & $11.2(13.8)$ & $6.7(11.1)$ & 0.013 & 0.41 \\
\hline
\end{tabular}

Table 3 shows the results of the UDI, DDI and IIQ domain scores before and after surgery. On all urogenital domains, there was significant improvement as well as on all quality of life domains. Symptoms on domain constipation and obstructive defecation also improved significantly. Large effect sizes were found on domain pain (effect size $=$ 0.92 ) and genital prolapse (effect size $=2.0$ ) of the UDI. The domain physical functioning and emotional health of the IIQ also showed a large effect size ( 0.82 and 0.79 , respectively).

In addition to the table we made a sub-analysis for the 15 women who had additional surgery for urinary incontinence (TVT). These women improved significantly on the urinary incontinence domain after surgery (mean score: $26.7 \rightarrow 6.7$, $p=0.009$ ). This improvement was not significant for the women without TVT surgery (mean score: $16.4 \rightarrow 11.5, p=$ 0.162 ). On the other hand, the women with TVT surgery did not improve significantly on the overactive bladder domain (mean score: $24.3 \rightarrow 16.23, p=0.079$ ) where the group without TVT did (mean score: $30.8 \rightarrow 14.3, p=0.000$ ).

\section{Discussion}

The objective of this study was to assess quality of life and urogenital and defecatory symptoms before and after sacrospinous hysteropexy. The results show that a sacrospinous hysteropexy significantly reduced all urogenital and several defecatory symptoms and significantly improved quality of life. Effect sizes were large on domain genital prolapse and pain (UDI), and on domain physical functioning and emotional health (IIQ). It also anatomically cured the uterine descent in $93.1 \%$ of women.

The sacrospinous ligament fixation was first described by Sederl (1958) [19]. Later it became more popular by
Richter and Albright [20] (Europe) and Randall and Nichols [21, 22] (USA). Several modifications of their techniques have been described since. The anatomical results of 2,256 women after a sacrospinous ligament fixation of the vaginal vault were recently reviewed [1]. Objective cure rates varied between 67 and $96.8 \%$, and subjective cure rates varied between 70 and $98 \%$. Our findings are in line with these results, although the review focused on the sacrospinous fixation of a vault prolapse. Subjective outcomes are underreported in most studies on the sacrospinous ligament fixation. We have shown prospectively that bladder and bowel function improves significantly after a sacrospinous hysteropexy.

There are a variety of reasons why women want to preserve their uterus. Among those reasons are: keeping their fertility, personal identity, but also the possibility that this kind of surgery might reduce operation time, estimated blood loss and postoperative recovery time $[4,23]$. There are signs that removing the uterus may increase the risk of pelvic neuropathy, new onset urinary incontinence, bladder dysfunction and prolapse [24, 25, 26]. Several studies on the sacrospinous hysteropexy, as a technique in which the uterus is preserved, are available [3-6, 8-10, 27]. Among these studies, three were of prospective design $[6,8,27]$, five were of retrospective design $[3-5,9,10]$ and there was one case report [28]. One report described a different surgical technique and therefore cannot be compared with our study [2]. One study assessed risk factors for failure of sacrospinous hysteropexy [29] and another study only assessed sexual functioning after sacrospinous hysteropexy [30]. Anatomic success rates in these studies varied between 74 and $93.5 \%$, which is comparable with our results. The main problems when comparing studies on the sacrospinous ligament fixation were recently debated by 
Morgan et al. [11]. They showed that there is a variety in definition of failure of sacrospinous ligament fixation due to differences in how anatomical outcomes are evaluated and which compartment of the vagina is considered. In our group, recurrent postoperative cystoceles, stage 2 or higher, were seen in 10 women $(13.9 \%)$. This percentage is slightly lower than the $21.3 \%$ reported in the recent review [11]. However, our follow-up in the current study was relatively short and some recurrences may not have been detected in this timeframe. In a previous retrospective study on the anatomical outcome of the sacrospinous hysteropexy by our group, we found a higher rate of recurrent cystoceles stage 2 or higher $(30 \%)$ [9]. The follow-up period in this study was a mean of 23 months. The high rate of recurrent cystoceles may be related to the primary damage of neuromuscular support or may be the result of the retroverted axis of the vagina after sacrospinous hysteropexy. This last aspect, being regarded as an overcorrection, is held responsible for the high rate of cystoceles [27, 31]. However, in a study by Smilen et al. [32] the sacrospinous hysteropexy did not independently increase the risk of recurrent cystocele as compared to other surgical techniques.

Apart from true genital prolapse symptoms, urogenital symptoms and also bowel symptoms improved after the sacrospinous hysteropexy. Because the majority of women in our study had their sacrospinous hysteropexy combined with an anterior colporrhaphy, one may argue that it was this anterior repair that relieved symptoms, and not the sacrospinous hysteropexy. However, it was shown that pelvic organ prolapse and urogenital symptoms were only slightly correlated to the site and severity of the prolapse [14]. This lack of a clear correlation between the site of the pelvic organ prolapse and symptomatology makes it very difficult in combination surgery to contribute functional improvement to a certain intervention. All we can conclude from our results is that surgical procedures that involve a sacrospinous hysteropexy show good functional outcome.

It was shown that overactive bladder symptoms disappear after anterior repair in $60-82 \%$ of women [33]. We also found a marked improvement of overactive bladder symptoms after surgery in our group. However, this significant improvement was confined to the women who did not have a combined TVT procedure with their sacrospinous hysteropexy. Women who did have a TVT combined procedure experienced more bother on overactive bladder domain after surgery as compared to women without TVT surgery. This finding is consistent with literature on the TVT in which the development of overactive bladder symptoms after TVT surgery is reported to occur in up to $15 \%$ of women [34].

We have to keep this in mind when placing a TVT (prophylactic) in case of occult stress incontinence. After sacrospinous hysteropexy, postoperative complications occur, but none of them was life threatening. Most complications were self-limiting. The majority of postoperative complications were related to the bladder function. These complications did not occur in women who only had a sacrospinous hysteropexy. Therefore, it is likely that complications related to the bladder are the consequence of additional surgery and not the result of sacrospinous hysteropexy. The prevalence of buttock pain is estimated at 10 to $15 \%$ [35]. This pain can be explained by injury to surrounding nerves of the sacral plexus and branches of the pudendal nerve. In an anatomical study, the relationship of the pudendal nerve to the sacrospinous ligament was found to be variable (one branch of the pudendal nerve piercing through the ligament was found in 11\%) [36]. Barksdale et al. also showed that nerve tissue is present and widely distributed within the sacrospinous ligament [37]. Therefore, although the placement of the suture two centimetres medial to the ischial spine protects against major nerve injuries, the complications of buttock pain cannot be prevented in all women. Fortunately, this buttock pain was shown to resolve spontaneously in most cases, as we also demonstrated in our series [9].

The strength of our study is that we measured urogenital and defecatory symptoms and quality of life in a large group of women who underwent a sacrospinous hysteropexy, with a validated questionnaire before and after surgery. There are some potential drawbacks that need to be discussed. First, there might be an indication bias. In our country, a vaginal hysterectomy is the standard surgical technique for correcting a uterine descent. Therefore, women that came to our hospital may have chosen specifically for this operation. They might have had high expectations of this procedure which could have influenced their outcome with respect to quality of life. Second, in some patients, follow-up time was limited to 3 months. Possibly, some recurrences had not yet developed at that time. Third, the study was performed in a single university hospital. The sacrospinous ligament fixation has become rapidly popular in our center and is performed by two surgeons. They are highly trained in performing the procedure. This might have influenced the outcome. Fourth, we did not compare the sacrospinous hysteropexy with another surgical technique to correct a uterine descent. Therefore, we cannot conclude that the sacrospinous hysteropexy is superior to other procedures. However, it is a safe and effective operation for women who wish to preserve their uterus at time of genital prolapse surgery.

Acknowledgements None.

Financial support/conflicts of interest None. 
Open Access This article is distributed under the terms of the Creative Commons Attribution Noncommercial License which permits any noncommercial use, distribution, and reproduction in any medium, provided the original author(s) and source are credited.

\section{References}

1. Beer M, Kuhn A (2005) Surgical techniques for vault prolapse: a review of the literature. Eur J Obstet Gynecol Reprod Biol 119 (2): $144-155$

2. Kovac SR, Cruikshank SH (1993) Successful pregnancies and vaginal deliveries after sacrospinous uterosacral fixation in five of nineteen patients. Am J Obstet Gynecol 168:1778-1786

3. Richardson DA, Scotti RJ, Ostergard DR (1998) Surgical management of uterine prolapse in young women. J Reprod Med 34(6):388-392

4. van Brummen HJ, van de PG, Aalders CI, Heintz AP, van der Vaart CH (2003) Sacrospinous hysteropexy compared to vaginal hysterectomy as primary surgical treatment for a descensus uteri: effects on urinary symptoms. Int Urogynecol $\mathrm{J}$ Pelvic Floor Dysfunct 14:350-355

5. Allahdin S, Herd D, Reid BA (2005) 25 Sacrospinous ligament fixation procedures in a district general hospital: our experience. J Obstet Gynecol 25(4):361-363

6. Hefni MA, El-Toukhy TA (2006) Long-term outcome of vaginal sacrospinous colpopexy for marked uterovaginal and vault prolapse. Eur J Obstet Gynecol Reprod Biol 127(2):257-263

7. Diwan A, Rardin CR, Kohli N (2004) Uterine preservation during surgery for uterovaginal prolapse: a review. Int Urogynecol $\mathrm{J}$ 15:286-292

8. Hefni MA, El Toukhy TA, Bhaumik J, Katsimanis E (2003) Sacrospinous cervicocolpopexy with uterine conservation for prolapse in elderly women: an evolving concept. AJOG 188 (3):645-650

9. Dietz V, de Jong J, Huisman M, Schraffordt Koops S, Heintz P, van der Vaart H (2007) The effectiveness of the sacrospinous hysteropexy for the primary treatment of uterovaginal prolapse. Int Urogynecol J Pelvic Floor Dysfunct (in press)

10. Maher CF, Cary MP, Slack MC, Murray CJ, Milligan M, Schluter P (2001) Uterine preservation or hysterectomy at sacrospinous colpopexy for uterovaginal prolapse? Int Urogynecol J 12:381-385

11. Morgan DM, Rogers MAM, Huebner M, Wei JT, DeLancy JO (2007) Heterogeneity in anatomic outcome of sacrospinous ligament fixation for prolapse. Obstet Gynecol 109(6):1424-1433

12. Ulmsten U, Hendriksson L, Johnson P, Vahros G (1996) An ambulatory surgical procedure under local anesthesia for treatment of female urinary incontinence. Int Urogynecol J Pelvic Floor Dysfunct 7(2):81-85

13. Bump RC, Mattiasson A, Bo K, Brubaker LP, Delancy JO, Klarskov P et al (1996) The standardisation of terminology of female pelvic organ prolapse and pelvic floor dysfunction. Am J Obstet Gynecol 175:10-17

14. Ellerkmann RM, Cundiff GW, Melick CF, Nihira MA, Leffler K, Bent AE (2001) Correlation of symptoms with location and severity of pelvic organ prolapse. Am J Obstet Gynecol 185 (6): $1332-1337$

15. van der Vaart CH, de Leeuw JR, Roovers JP, Heintz AP (2003) Measuring health-related quality of life in women with urogenital dysfunction: the urogenital distress inventory and incontinence impact questionnaire revisited. Neurourol Urodyn 22:97-104

16. Roovers JP, van der Bom JG, van der Vaart CH, Heintz AP (2005) Prediction of findings at defecography in patients with genital prolapse. BJOG 112(11):1547-1553
17. van Brummen HJ, Bruinse HW, van de Pol G, Heintz AP, van der Vaart CH (2006) Defecatory symptoms during and after the first pregnancy: prevalences and associated factors. Int Urogynecol J Pelvic Floor Dysfunct 17(3):224-230

18. Cohen J (1988) Statistical power analysis for the behavioural sciences, 2nd edn. Erlbaum, Hillsdale NY

19. Sederl J (1958) Zur operation des prolapses der blind endigenden sheiden. Geburtshilfe Frauernheilkd 18:824-828

20. Richter K, Albright W (1981) Long term results following fixation of the vagina on the sacrospinous ligament by the vaginal route. Am J Obstet Gynecol 141:811-816

21. Randall CL, Nichols DH (1971) Surgical treatment of vaginal eversion. Obstet Gynecol 38:327-332

22. Nichols DH (1982) Sacrospinous fixation for massive eversion of the vagina. Am J Obstet Gynecol 142:901-904

23. Thomas AG, Brodman ML, Dottine PR, Bodian C, Friedman F, Bogursky L (1995) Manchester procedure vs. vaginal hysterectomy for uterine prolapse. J Reprod Med 40:299-304

24. Nesbitt RE (1989) Uterine preservation in the surgical management of genuine stress urinary incontinence associated with uterovaginal prolapse. Surg Gynecol Obstet 1989 168:143-147

25. Kjerluff KH, Langenberg PW, Greenaway L, Uman J, Harvey LA (2002) Urinary incontinence and hysterectomy in a large prospective cohort study in American women. J Urol 167:2088-2092

26. Petros PE (2000) Influence of hysterectomy on pelvic-floor dysfunction. Lancet 356:1275

27. Carey MP, Slack MC (1994) Transvaginal sacrospinous colpopexy for vault and marked uterovaginal prolapse. Br J Obstet Gynaecol 101:536-540

28. Hefni M, El-Toukhy T (2002) Sacrospinous cervico-colpopexy with follow-up 2 years after successful pregnancy. Eur J Obstet Gynecol Reprod Biology 103(2):188-190

29. Lin TY, SU TH, Lee MY, Hsieh CH, Wang KG, Chen GD (2005) Risk factors for failure of transvaginal sacrospinous uterine suspension in the treatment of uterovaginal prolapse. J Formos Med Assoc 104(4):24-53

30. Jeng CJ, Yang YC, Tzeng CR, Shen J, Wang LR (2005) Sexual functioning after vaginal hysterectomy or transvaginal sacrospinous uterine suspension for uterine prolapse: a comparison. J Reprod Med 50(9):669-674

31. Colombo M, Milani R (1998) Sacrospinous ligament fixation and modified McCall culdoplasty during vaginal hysterectomy for advanced uterovaginal prolapse. Am J Obstet Gynecol 179(1):1320

32. Smilen SW, Saini J, Wallach SJ, Porges RF (1998) The risk of cystocele after sacrospinous ligament fixation. Am J Obstet Gynecol 179(6 Pt 1):1465-1471

33. Digesu GA, Salvatore S, Chaliha C, Athanasiou S, Milani R, Khullar V (2007) Do overactive bladder symptoms improve after repair of anterior vaginal wall prolapse? Int Urogynecol J Pelvic Floor Dysfunct 18(12):1439-1443

34. Abouassaly R, Steinberg JR, Lemieux M, Marois C, Gilchrist LI, Bourque JL, Tu le M, Corcos J (2004) Complications of tensionfree vaginal tape surgery: a multi-institutional review. BJU 94 (1):110-113

35. Karram MM, Walters MD (1993) Pelvic organ prolapse: enterocele and vaginal vault prolapse. Mosby-yearbook, St Louis, pp 245-252

36. Mahakkanukrauh P, Surin P, Vaidhayakarn P (2005) Anatomical study of the pudendal nerve adjecent to the sacrospinous ligament. Clin Anat 18(3):200-205

37. Barksdale PA, Gasser RF, Gauthier CM, Elkins TE, Wall LL (1997) Intraligamentous nerves as a potential source of pain after sacrospinous ligament fixation of the vaginal apex. Int Urogynecol J Pelvic Floor Dysfunct 8(3):121-125 